\title{
Crystal collimator systems for high energy frontier
}

\author{
A. I. Sytov, ${ }^{*}$ V. V. Tikhomirov, and A. S. Lobko \\ Institute for Nuclear Problems, Belarusian State University, \\ Bobruiskaya street, 11, Minsk 220030, Belarus \\ (Received 9 July 2016; published 13 July 2017)
}

\begin{abstract}
Crystalline collimators can potentially considerably improve the cleaning performance of the presently used collimator systems using amorphous collimators. A crystal-based collimation scheme which relies on the channeling particle deflection in bent crystals has been proposed and extensively studied both theoretically and experimentally. However, since the efficiency of particle capture into the channeling regime does not exceed ninety percent, this collimation scheme partly suffers from the same leakage problems as the schemes using amorphous collimators. To improve further the cleaning efficiency of the crystal-based collimation system to meet the requirements of the FCC, we suggest here a double crystalbased collimation scheme, to which the second crystal is introduced to enhance the deflection of the particles escaping the capture to the channeling regime in its first crystal. The application of the effect of multiple volume reflection in one bent crystal and of the same in a sequence of crystals is simulated and compared for different crystal numbers and materials at the energy of $50 \mathrm{TeV}$. To enhance also the efficiency of use of the first crystal of the suggested double crystal-based scheme, we propose: the method of increase of the probability of particle capture into the channeling regime at the first crystal passage by means of fabrication of a crystal cut and the method of the amplification of nonchanneled particle deflection through the multiple volume reflection in one bent crystal, accompanying the particle channeling by a skew plane. We simulate both of these methods for the $50 \mathrm{TeV}$ FCC energy.
\end{abstract}

DOI: 10.1103/PhysRevAccelBeams.20.071001

\section{INTRODUCTION}

The collimation system is of key importance for stable operation of modern high energy storage rings and colliders, for instance for the LHC and the future HL-LHC project [1-2]. Moreover, "the operation and physics goals of recent superconducting, high-energy hadron colliders, such as the Tevatron, the Relativistic Heavy-Ion Collider (RHIC), and the Large Hadron Collider (LHC), could not be fulfilled without adequate beam collimation" [3]. The small-angle scattering in collimators is used to induce a steady betatron amplitude growth resulting in particle collisions with the target collimators (absorbers) before they reach the apertures of superconducting magnets. Thick amorphous collimators are used at the LHC, RHIC and SPS at present. The efficiency of any collimation scheme is limited by the leakage to sensitive equipment. Since the uncorrelated Coulomb scattering in the amorphous collimators is characterized by the small average scattering angles, the leakage of the collimation schemes with amorphous collimators is determined by both the multiple particle passages through the latter and the small impact

*alexei.sytov@cern.ch

Published by the American Physical Society under the terms of the Creative Commons Attribution 3.0 License. Further distribution of this work must maintain attribution to the author $(s)$ and the published article's title, journal citation, and DOI. parameters of particle collisions with the target collimators (absorbers).

Crystal collimators can be used to decrease the leakage of the present collimation systems [3-4]. The point is that a bent crystal can deflect channeling particles by the angles large enough to hit target collimators immediately and with a large impact parameter. Such a collimator scheme, using planar channeling in bent crystals, proposed in [5], has been widely explored in experiments [6-15].

However, this collimation approach also suffers from the leakage. The latter is mainly induced by more than ten percent of the particles inevitably escaping the capture to the channeling regime at their first passage through the crystal collimator. As with the amorphous collimators, many of these particles reach target collimators by means of volume reflection [16] or Coulomb scattering only after a number of passages through the crystal collimator having small impact parameters. That is why further possibilities to improve the efficiency of the crystal assisted collimation system still remains.

Therefore, we suggest a new double crystal-based collimation system, as shown in Fig. 1. This layout represents the FCC betatron cleaning insertion with betafunctions and absorber transverse positions (12.6 $\sigma$ ) taken from [17]. In the trajectories simulated only betatron oscillations have been taken into account, while the synchrotron ones have been neglected because of too low values of the dispersion functions. 


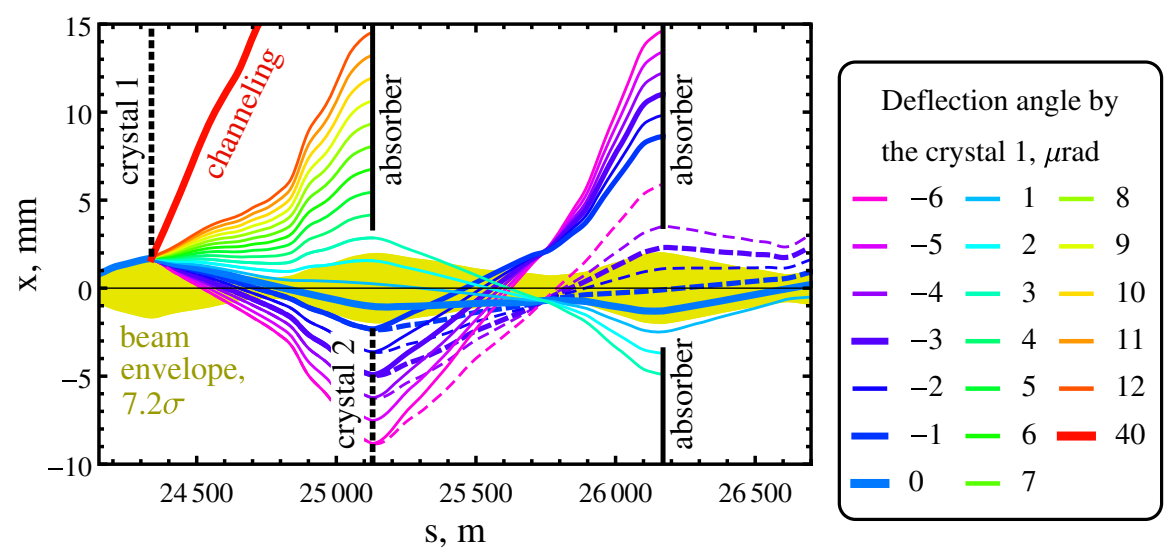

FIG. 1. Double crystal-based collimation system. Beam trajectories for different deflection angles by the crystal 1 are simulated in the betatron cleaning area of the FCC [17], taking into account only betatron oscillations. The crystal 1, crystal 2 and the absorbers are placed at $7.2 \sigma, 9 \sigma$ and $12.6 \sigma$ respectively. The particles, passed through the crystal 2 , are assumed to be deflected by $-5 \mu$ rad for solid lines and $0 \mu \mathrm{rad}$ for the dashed ones. Bold curves illustrate the trajectories of non-deflected particle, of channeled particles and particles deflected by 1 and $3 \mu \mathrm{rad}$, being the typical angles of particle deflection by volume reflection (VR) and multiple volume reflection in one bent crystal (MVROC) respectively.

The bent crystal 1 is placed at $7.2 \sigma$, i.e. at the transverse position of primary collimators [17], deflecting most of the particles into the absorber in the channeling mode. The second crystal, placed at $\sim 9 \sigma$, plays role of the secondary collimator intercepting particles, volume reflected in the crystal 1 , and deflecting them into the absorber.

The collimation system proposed demonstrates the following advantages. 1) The impact parameters of particles at the absorbers are large enough to prevent the leakage at the absorber boundary. 2) Close to $100 \%$ of halo particles will be intercepted by only one passage through the betatron cleaning insertion.

We propose different coherent effects, being promising for this application in both bent crystals. In particular, for the second crystal we suggest the several-fold increase of the typical angle of scattering and volume reflection in the crystal collimator by the effect of multiple volume reflection in one bent crystal (MVROC) [18-19]. A good alternative to MVROC can be volume reflection in a sequence of crystals [20]. The main advantage of both effects is large angular acceptance to deflect coherently almost all the particles passing through the crystal and large enough deflection angle to deflect the particles into the absorber by few or even only one crystal passage. It is necessary to underline that both of effects have been already observed in experiments in concordance with theory [20-23].

To amplify the deflection angle even more, we propose to use the advantages of both of these effects by using of MVROC in a sequence of crystals. Moreover we study MVROC in crystals of different materials: silicon, germanium and tungsten. We optimize the parameters of the crystal as well as its alignment for each effect both theoretically and by means of Monte Carlo simulations on example of $50 \mathrm{TeV}$ protons of Future Circular Collider (FCC) [24-27]. We compare these effects by the peak deflection angle.
The second approach is the application of planar channeling in skew crystal planes [28] instead of the vertical ones. Its main advantage is deflection of nonchanneled particles by means of MVROC instead of volume reflection considerably amplifying the deflection angle. The angular acceptance and, consequently, the channeling efficiency also increases. In addition, we suggest to considerably increase the channeling efficiency by application of the crystal cut $[29,30]$.

Combination of these two approaches can lead to the further collimation optimization. Each of suggested coherent effects has a potential to considerably increase the efficiency of the crystal-based collimation system.

\section{VOLUME REFLECTION IN A SEQUENCE OF BENT CRYSTALS}

Volume reflection at optimal conditions provides a deflection angle $\alpha$ of approximately $\alpha \approx 1.5 \theta_{L}$ [31], where $\theta_{L}=\sqrt{2 U_{0} / p v}$ is the critical channeling angle (Lindhard angle) [32], $U_{0}$ is the height of interplanar potential well, $p$ is the momentum of a particle and $v$ is its velocity. More exact calculations of the ratio of the volume reflection angle to the Lindhard angle $\theta_{L 0}$ (for straight crystal) can be provided by a formula from [31] and rewritten, taking into account the critical radius $R_{c r}=p v / E_{0}$, where $E_{0}$ is the maximal strength of interplanar electric field:

$$
\begin{aligned}
\frac{\alpha}{\theta_{L 0}}= & \frac{E_{0}}{\eta \sqrt{U_{0}}} \int_{x_{0}}^{x_{c}(\epsilon)}\left[\frac{1}{\sqrt{\epsilon-U(x)-\frac{E_{0}}{\eta} x}}\right. \\
& \left.-\frac{1}{\sqrt{\epsilon-U\left(x_{c}(\epsilon)\right)-\frac{E_{0}}{\eta} x}}\right] d x .
\end{aligned}
$$

Here $\eta$ is the ratio of the bending radius $R$ to its critical value $R_{c r}, U(x)$ is the interplanar potential function of a 


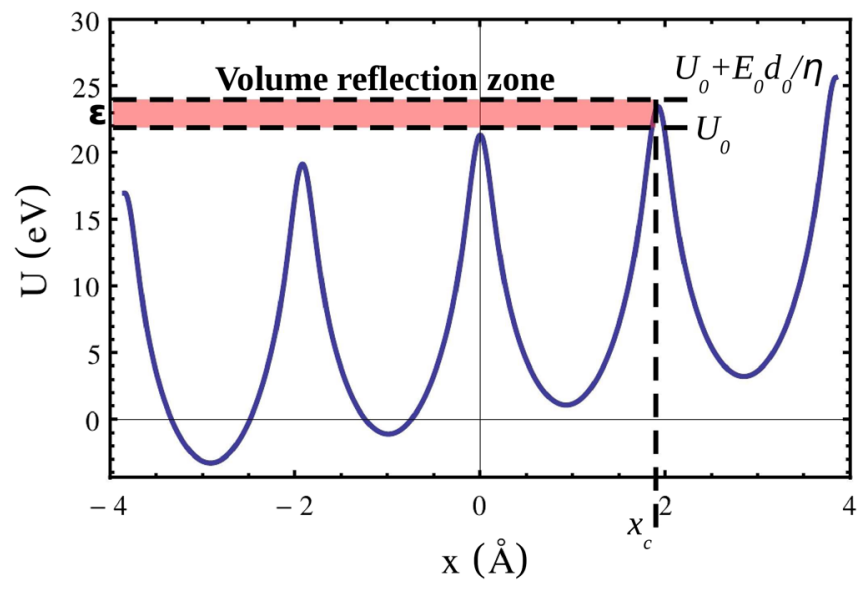

FIG. 2. Interplanar potential in (110) Si crystal.

straight crystal on transverse coordinate $x, \frac{E_{0}}{\eta} x$ represents centrifugal fraction of interplanar potential, $\epsilon$ is the transverse energy, $x_{0}$ is the initial coordinate of particle motion, and $x_{c}$ is the volume reflection point (see Fig. 2). The dependence on transverse energy can be reduced by its averaging on over-barrier values, the distribution of which can be approximately considered as uniform [31]:

$$
\left\langle\frac{\alpha}{\theta_{L 0}}\right\rangle=\frac{\eta}{E_{0} d_{0}} \int_{U_{0}}^{U_{0}+\frac{E_{0}}{\eta} d_{0}} \frac{\alpha}{\theta_{L 0}} d \epsilon,
$$

where $d_{0}$ is the interplanar distance. For the simplicity we will use this formula only for equidistant crystal planes in this paper, but it can be simply generalized on nonequidistant case. It should be noted that multiple scatterings have not been considered yet, because our goal is to estimate optimal parameters for Monte Carlo simulations.

Thus, the obtained formulas (1)-(2) depend only on interplanar potential and bending radius. They are very useful, because they do not depend on particle energy and therefore, can be verified with experiments already done as well as can be applied at the FCC energy.

A plot representing the dependence by Eqs. (1)-(2) for (110) planes is shown in Fig. 3.

Indeed, the maximal deflection angle of $1.53 \theta_{L 0}$ is reached at the bending radius

$$
R \approx 100 R_{c r}
$$

However, in reality $R=l_{c r} / \varphi$ is limited by the bending angle $\varphi$, to be at least several higher than $\theta_{L}$ and the crystal length $l_{c r}$, always limited by experimental reasons. These constraints are especially important for considering of volume reflections in a of bent crystals sequence (see Fig. 4).

All of the crystals in a sequence as well as their orientation are considered to be the same. Therefore, the sum of deflection angles at each crystal from Eq. (2) should

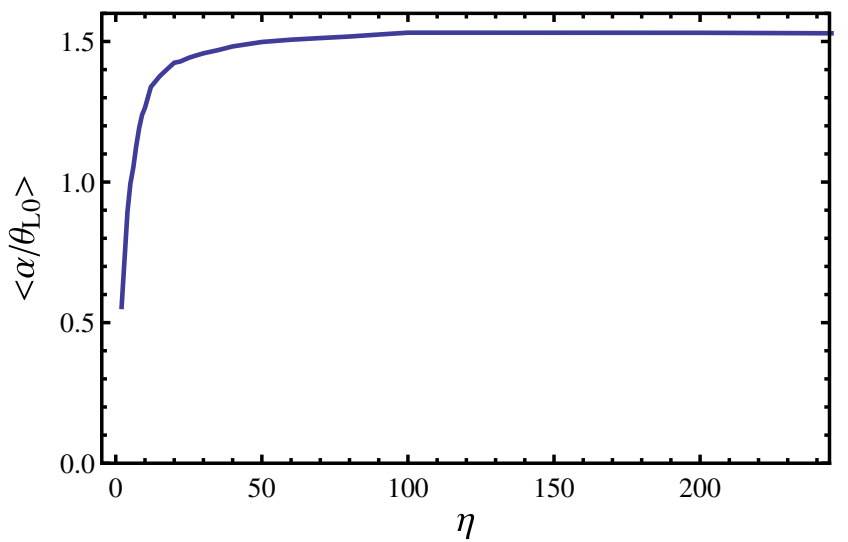

FIG. 3. The dependence of the ratio of volume reflection angle to the Lindhard angle on the ratio of the bending radius to its critical value.

be less than the bending angle. Moreover, to avoid the capture into the channeling mode, the angle of alignment of any crystal in a sequence should be at least on $1.5 \theta_{L 0}$ less than the current beam direction. This can be transformed into the condition:

$$
n\left\langle\frac{\alpha}{\theta_{L 0}}\right\rangle(\eta)=\varphi-3 \theta_{L 0},
$$

where $n$ is a number of bent crystals in a sequence, volume reflection angle is fixed with respect to the interplanar potential. We fix also the total length of the sequence $L_{\text {seq }}$, because, for instance, for the crystal-based collimation it is optimized taking into account constraints on inelastic nuclear scattering rate [33]. It allows one to calculate the bending angle as:

$$
\varphi=L_{\mathrm{seq}} / n \eta R_{c r}
$$

transforming the condition (4) into:

$$
n\left\langle\frac{\alpha}{\theta_{L 0}}\right\rangle(\eta)=L_{\text {seq }} / n \eta R_{c r}-3 \theta_{L 0}
$$

and expressing $\eta$, one can find an optimal bending radius as a function of $n$. Unfortunately, Eq. (6) is not linear, but one can simply solve it numerically.

We applied this equation to calculate the optimal parameters of a sequence of silicon crystals for different length values: $2.5 \mathrm{~cm}, 5 \mathrm{~cm}, 7.5 \mathrm{~cm}$ and $10 \mathrm{~cm}$ and for different number of crystals. These parameters were used for Monte Carlo simulations with application of the

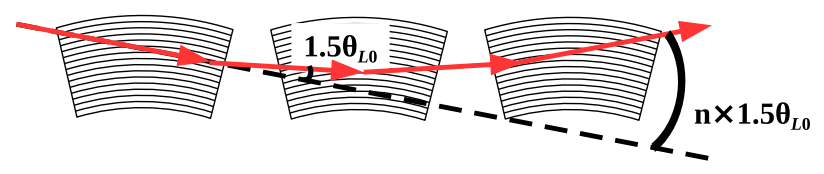

FIG. 4. Volume reflection in a sequence of bent crystals. 

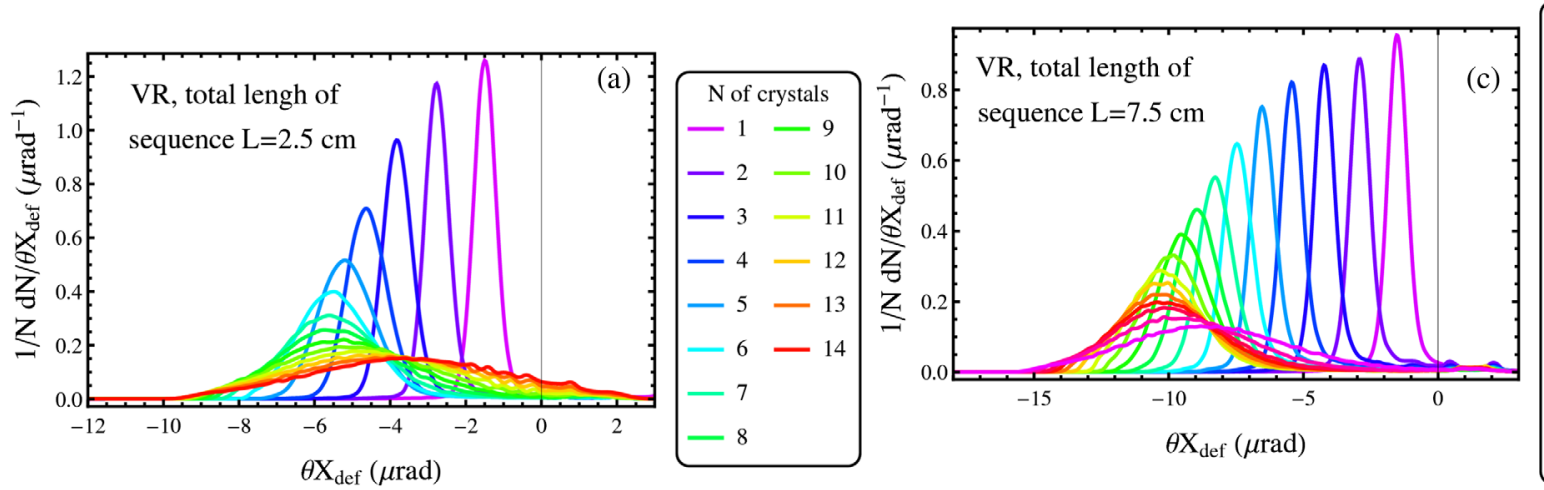

\begin{tabular}{|cc|}
\hline \multicolumn{2}{|c}{ of crystals } \\
-1 & -11 \\
-2 & -12 \\
-3 & -13 \\
-4 & -14 \\
-5 & -15 \\
-6 & -16 \\
-7 & -17 \\
-8 & \\
-9 & \\
-10 & \\
\hline $\mathrm{N}$ of crystals \\
1 & -11 \\
-2 & -12 \\
-3 & -13 \\
4 & -14 \\
-5 & -15 \\
6 & -16 \\
7 & -17 \\
-8 & \\
9 & \\
-10 & \\
\hline
\end{tabular}
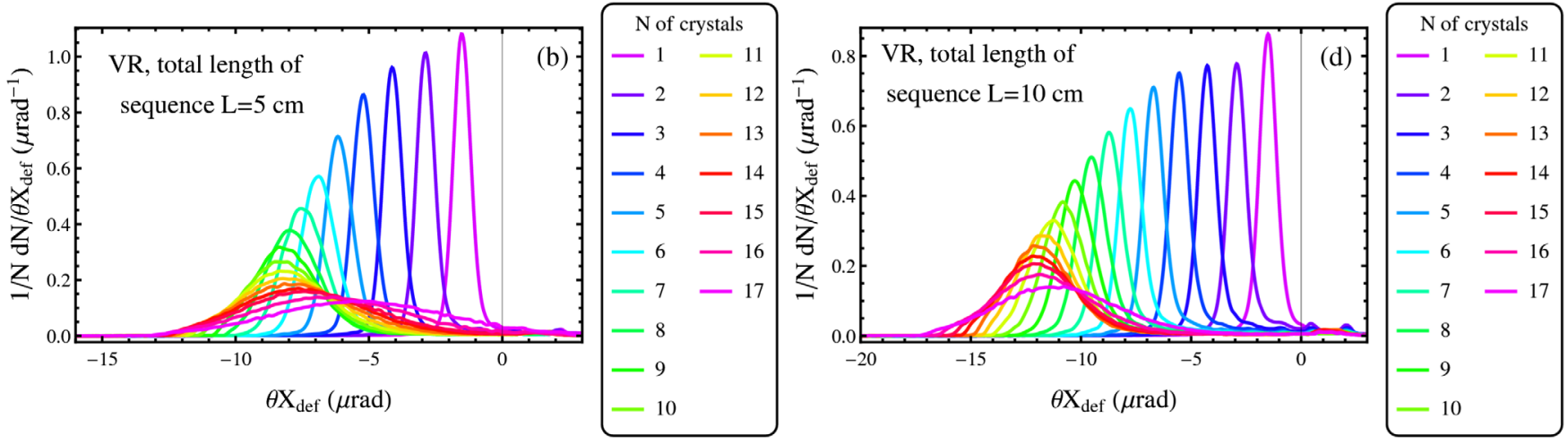

FIG. 5. Angular distribution behind the crystal for volume reflection of $50 \mathrm{TeV}$ protons in a sequence of (110) bent silicon crystals. Bending radius is calculated according to (6).

CRYSTAL simulation code [34-35]. The simulation results for $50 \mathrm{TeV}$ protons deflection with a statistics of 200000 particles are shown in Fig. 5. Hereinafter the initial angular divergence is taken at $2.3 \mu \mathrm{rad}$ which is 4 times lower than $\theta_{L 0}$ at $50 \mathrm{TeV}$ and more than an order lower than the angular acceptance of volume reflection and MVROC.

The results confirmed the existence of the optimal number of crystals depending on the sequence length. If the number of crystals is too low, the deflection angle is also low. However, for too high crystal number the peak deflection angle decreases, and the divergence of the deflected beam becomes too wide. This is mainly explained by a sharp decrease of deflection angle for small $R$ (see Fig. 3 ) and by increase of over-barrier transverse energy range [see Eq. (2) and Fig. 2], spreading deflection angles stronger.

Both the peak deflection angle and the optimal number of crystals increases with the rise of $L_{\mathrm{seq}}$. At $L_{\mathrm{seq}}=10 \mathrm{~cm}$ the peak deflection angle achieves $12 \mu \mathrm{rad}$ for 14 crystals in a sequence. However, depending on the experimental set up one can reduce the number of the crystals to decrease the spread of deflected beam.

\section{MULTIPLE VOLUME REFLECTION IN ONE BENT CRYSTAL AND IN A SEQUENCE OF BENT CRYSTALS}

The idea of multiple volume reflection in one bent crystal is volume reflections from skew crystal planes as shown in
Fig. 6. While in vertical direction these reflections almost compensate each other in horizontal one they will be summarized. For $\langle 111\rangle$ silicon crystal axes MVROC deflection angle for protons is 5 times higher than the volume reflection one [18-19], which was confirmed experimentally [21-23].

The optimal conditions of MVROC are mainly defined by horizontal $\theta_{x}$ and vertical $\theta_{y}$ angles of crystal alignment and bending angle $\varphi$ and crystal length $l_{c r}$.

The first condition is that both angles of crystal alignment should be at least 3-4 times higher on module than the axial critical angle [32] $\left(\theta_{y} \approx 4 \theta_{\text {Lax }} \approx 8 \mu \mathrm{rad}\right.$ at $\left.50 \mathrm{TeV}\right)$.

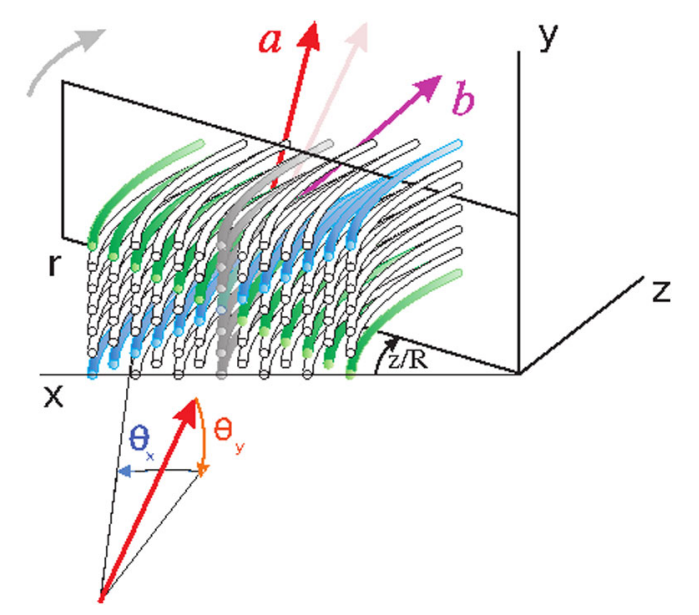

FIG. 6. Multiple volume reflection in one bent crystal. 
The second condition establishes the crystal orientation for the beam to be volume reflected by the strongest skew plane ((110) for $\langle 111\rangle$ axes). The inclination angle of the plane should exceed the angle of the plane of particles incidence to the crystal: $\alpha_{\text {inc }}>\arctan \left(\theta_{y} / \theta_{x}\right)$ for a plane to reflect particles [18-19]. For $\langle 111\rangle$ axes it will be transformed into condition:

$$
\theta_{x}<\theta_{y} \sqrt{3}
$$

in which the sign $>$ is changed to $<$ because angles of crystal alignment are negative. To avoid capture into the channeling mode $\theta_{x}$ should be smaller at least on 1-1.5 $\theta_{\mathrm{Lax}}$. For our case we have chosen $\theta_{x}=-17.5 \mu \mathrm{rad}$ and $\theta_{y}=-8 \mu \mathrm{rad}$.

The third condition determines the bending angle for the beam to be reflected by all main crystal planes. By the vertical symmetry of the crystal lattice this angle should be twice larger on the module than the horizontal incidence angle:

$$
\varphi=-2 \theta_{x}
$$

giving in our case $\varphi=35 \mu \mathrm{rad}$.

The optimal length of the crystal can be obtained from the condition of optimal $R$ for single volume reflection (3). However, it is limited by experimental conditions, and therefore should be as high as possible.
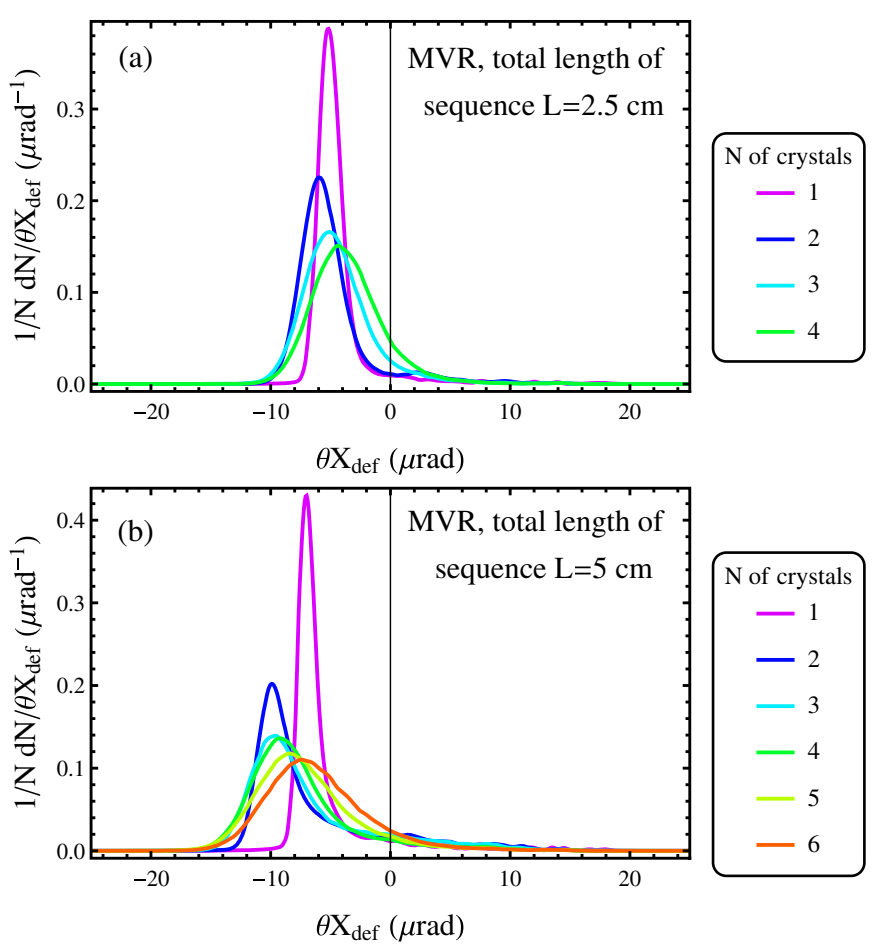

For the amplification of the deflection angle we propose to apply MVROC in a sequence of crystals similarly to the technique from the previous section. In a such setup it is very important to avoid capture in the channeling mode by skew crystal planes. Thereby, the condition (7) should be fulfilled for all the crystals in the sequence. Therefore, the angle of crystal alignment with respect to deflected beam by all the crystals except the last one $\theta_{x f}$ should also fulfill this condition:

$$
\theta_{x f}<\theta_{y} \sqrt{3}
$$

The difference between $\theta_{x f}$ and $\theta_{x}$ is equal to the deflection angle by all the crystals except the last one $\theta_{x f}-\theta_{x}=$ $(n-1) \alpha_{\text {MVROC }}$. The angle of deflection by means of MVROC $\alpha_{\text {MVROC }}$ can be estimated as 5 angles of single volume reflection [18,19,21], calculated above by Eqs. (1)-(2), giving:

$$
\theta_{x f}-\theta_{x}=5(n-1) \theta_{L 0}\left\langle\frac{\alpha}{\theta_{L 0}}\right\rangle(\eta)
$$

The angle $\theta_{x}$ can be defined by the condition (8). However, it is different for any crystal because of deflections. We suggest to use the average value of the crystal alignment for the first and the last crystal in a sequence $\frac{\theta_{x}+\theta_{x f}}{2}$ transforming the condition (8) into:
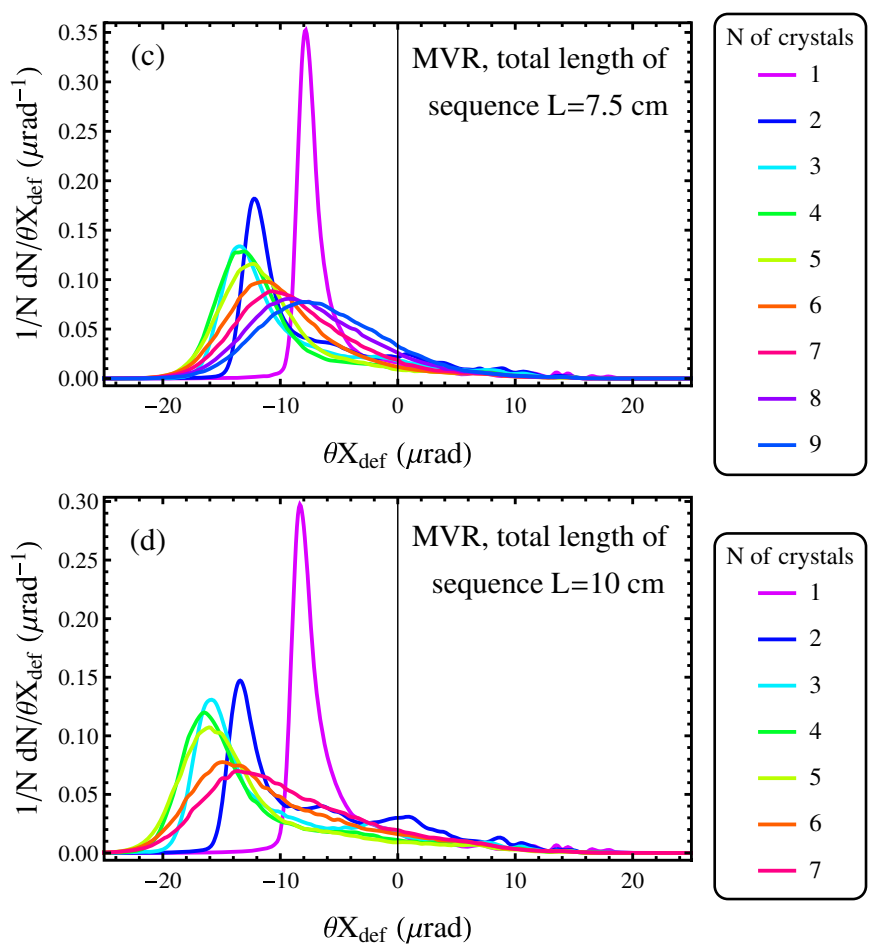

FIG. 7. Angular distribution behind the crystal for MVROC of $50 \mathrm{TeV}$ protons in one and in a sequence of $\langle 111\rangle$ bent silicon crystals. The alignment and geometry of crystals are calculated according to (7)-(12). 


$$
\varphi=-\theta_{x}-\theta_{x f} .
$$

Expressing $\varphi$ from Eq. (5), $\theta_{x}$ from Eq. (11) and substituting it into (10), one obtains an equation for the variable $\eta$ for MVROC analogically to Eq. (6):

$$
2 \theta_{x f}+L_{\mathrm{seq}} / n \eta R_{c r}=5(n-1) \theta_{L 0}\left\langle\frac{\alpha}{\theta_{L 0}}\right\rangle(\eta) .
$$

The angles $\theta_{x f}$ and $\theta_{y}$ are calculated by the same way as for the case of a single crystal. However, we lowed them a bit: $\theta_{x f}=-14 \mu \mathrm{rad}$ and $\theta_{y}=-7 \mu \mathrm{rad}$ to decrease the bending angle and to increase the deflection angle.

Analogical simulations to that, shown in Fig. 5, were conducted for MVROC both in a single crystal and in a sequence of silicon crystals with application of CRYSTAL simulation code [34-35] with the statistics of 200000 particles. The results are presented in Fig. 7. The initial parameters were optimized according to (7)-(12).

One can conclude that the optimal number of crystals varies from 2 to 3-4 for the sequence length from $2.5 \mathrm{~cm}$ to $10 \mathrm{~cm}$. This is considerably less than the number of the crystals for single volume reflection case. However, MVROC in a sequence of crystals provides considerably higher deflection angle than single volume reflection, $16.5 \mu \mathrm{rad}$ and $12 \mu \mathrm{rad}$ respectively for $L_{\mathrm{seq}}=10 \mathrm{~cm}$. The comparison of these cases and the case of MVROC only in one crystal (the peak angle is $8.3 \mu \mathrm{rad}$ ) is shown in Fig. 8.

In this figure simulations of MVROC in crystals of other materials are also presented, in particular in germanium and tungsten crystals. The atomic strings in crystals of heavy elements possess higher field deflecting particles on higher angles. However, the length of such crystals should be reduced in comparison with silicon to reach the same probability of nuclear inelastic interactions. Anyway they provide larger deflection angles with respect to silicon

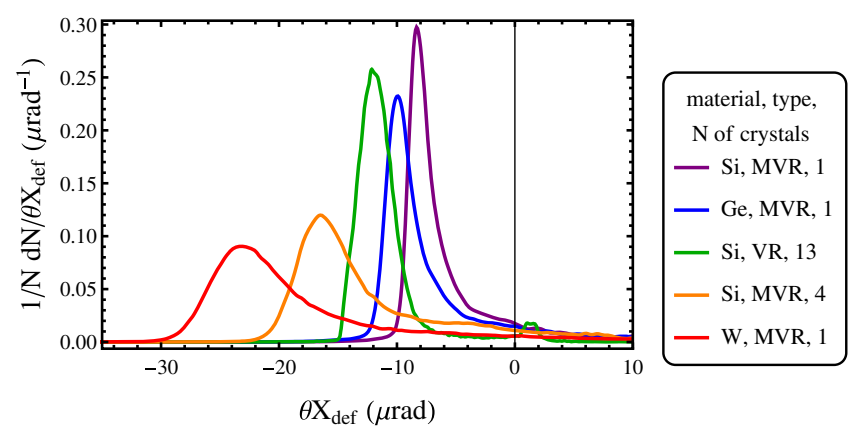

FIG. 8. Comparison of angular distribution behind the crystal for volume reflection and MVROC of $50 \mathrm{TeV}$ protons in one and a sequence of bent silicon crystals and MVROC in bent germanium and tungsten crystals. The length is $10 \mathrm{~cm}$ of the silicon crystal or crystal sequence, $5.4 \mathrm{~cm}$ of the germanium crystal and $1.9 \mathrm{~cm}$ of the tungsten crystal. crystals. In particular, the peak deflection angle for $50 \mathrm{TeV}$ protons reaches $23 \mu \mathrm{rad}$ for tungsten crystal. It 1.4 times exceeds the angle of MVROC in a sequence of Si crystals and twice exceeds the deflection angle of volume reflection in a sequence. Moreover, potentially the technique MVROC in a sequence can be applied also for tungsten crystals which can double the deflection angle. All of this can be applied for the crystal-based collimation system, because the deflection angles for almost all the particles exceed 3-5 $\mu \mathrm{rad}$. It is enough for particle deflection by the crystal 2 into the absorber with high impact parameters a shown in Fig. 1.

\section{CHANNELING IN SKEW CRYSTAL PLANES AND A CRYSTAL WITH A CUT}

There is a possibility to combine the advantages of channeling and MVROC [28]. For this we suggest to align the crystal for channeling in skew crystal planes instead to avoid it as in the previous section. In this case the inequality (7) become equality, changing the optimal crystal orientation only in x-plane. However, the (110) skew plane with a $\alpha_{\text {inc }}=30^{\circ}$ is not very useful because provides too high angle in vertical plane and too low in the horizontal one. To double the latter we propose to rotate the crystal lattice on $30^{\circ}$ as shown in Fig. 9. The condition (7) will be rewritten as:

$$
\theta_{x}=\theta_{y} / \tan \alpha_{\text {inc }}=\theta_{y} / \sqrt{3} .
$$

To reach the same deflection angle as for channeling in vertical planes, one should bend the crystal stronger $1 / \sin ^{2} \alpha_{\text {inc }}$ times. However, the channeling efficiency will not decrease. Moreover, channeling in skew crystal planes provides higher angular acceptance increasing the channeling efficiency. This is confirmed by our simulations (with CRYSTAL simulation code [34-35] with a statistics of $10^{6}$ particles, presented in Fig. 10. The efficiency of channeling in skew crystal planes reaches $80.5 \%$, while for vertical planes it is $79 \%$.

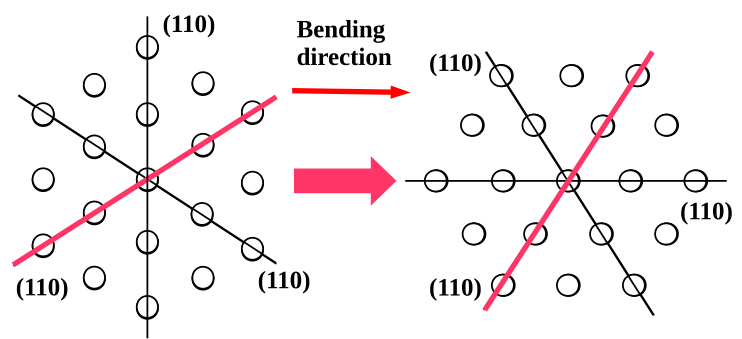

FIG. 9. Rotation of crystal lattice for application of channeling in skew planes. In both case crystal is bent horizontally. Red line indicates the plane (110) used for channeling. 


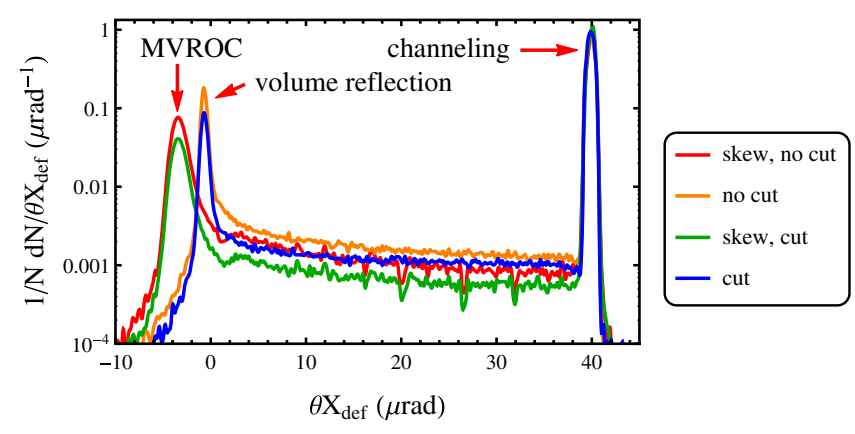

FIG. 10. The deflection angle distribution behind a bent crystal of $5 \mathrm{~cm}$ long for planar channeling in vertical and skew (110) crystal planes of $50 \mathrm{TeV}$ protons. The bending angle it $40 \mu \mathrm{rad}$ and $53.3 \mu \mathrm{rad}$ for vertical and skew crystal planes respectively. The parameters of crystal cut are calculated according to [29].

However, the main advantage of channeling in skew crystal planes is deflection of nonchanneled particles by means of MVROC instead of volume reflection. MVROC deflects particles at the angle of $3.3 \mu \mathrm{rad}$ which is 5 times stronger than volume reflection. This is very important for the crystal-based collimation system, because small deflection angle causes more additional passages of particles through the crystal and, consequently, increases the inelastic nuclear scattering rate. In opposite, the angles of MVROC are high enough to redirect almost all nonchanneled particles onto the crystal 2 as shown in Fig. 1.

There is a possibility to increase the channeling efficiency in both cases by means of a narrow plane cut [29-30] see Fig. 11. The idea of this technique is determined by similarity of phases in the channeling mode at the crystal entrance. Therefore, one can focus synphasely particles in the cut to the center of the channel. Unfortunately, it works efficiently only for the beam angular divergence less than a quarter of the $\theta_{L 0}$ and requires a very exact crystal alignment of the same order. Anyway, in our case (see Fig. 10) it allows one to increase the channeling efficiency up to $87.5 \%$ for usual planar channeling and up to $89 \%$ for channeling in skew planes.

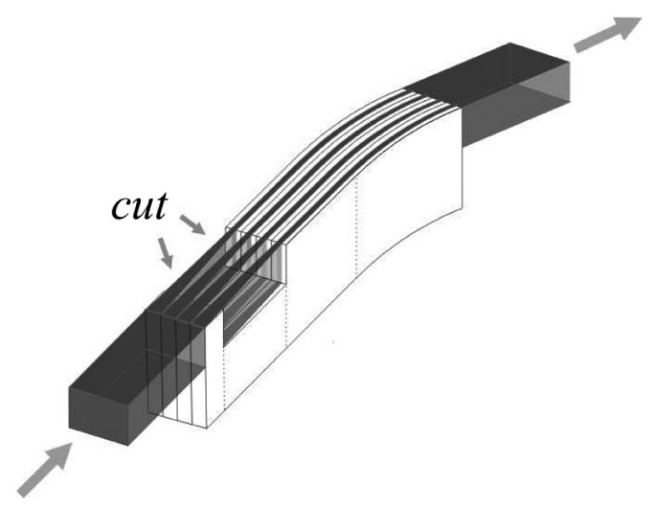

FIG. 11. A bent crystal with a cut.

\section{BETATRON CLEANING INSERTION}

The main function of the collimation system as well as of coherent effects proposed is to intercept as many particles as possible during only one passage through betatron cleaning insertion. Therefore, a considerable decrease of non-intercepted particles fraction with respect to the standard (single) crystal-based collimation scheme will testify an advantage of the new collimation scheme.

In order to verify this approach we used the crystal-based collimation scheme described in Fig. 1. For simulations four variants of the collimation scheme were chosen: (1) single collimation scheme with the first crystal, bent along (110) vertical planes, and with an absorber at $12.6 \sigma$ instead of the second crystal; (2) the same with the first crystal with a cut; (3) double crystal-based collimation scheme with the first crystal, aligned for channeling along (110) skew crystal planes, formed by $\langle 111\rangle$ axes, and the second one, designed for the MVROC effect; (4) the combination of (2) and (3). The parameters of the first crystal are the same as in simulations, presented in Fig. 10, i.e., a $5 \mathrm{~cm}$ silicon crystal, with the bending angle of $40 \mu \mathrm{rad}$ for vertical and $53.3 \mu \mathrm{rad}$ for skew (110) crystal planes, ideally aligned for the channeling mode. The second crystal is the same as used in simulations, shown in Fig. 8, namely a $10 \mathrm{~cm}$ silicon crystal, with the bending angle of $35 \mu \mathrm{rad}$.

The beam distribution at the first crystal entrance, generated using the diffusion model [36], is shown in Fig. 12. The angular divergence, being approximately $0.04 \mu \mathrm{rad}$, is considerably lower than the Lindhard angle. The Future Circular Collider parameters [17,24-27,37] were used for all our simulations. The simulations were carried out by CRYSTAL simulation code [34-35] with a statistics of $10^{6}$ particles in each case, taking into account both betatron and synchrotron oscillations. The absorbers are assumed to intercept all the incident particles.

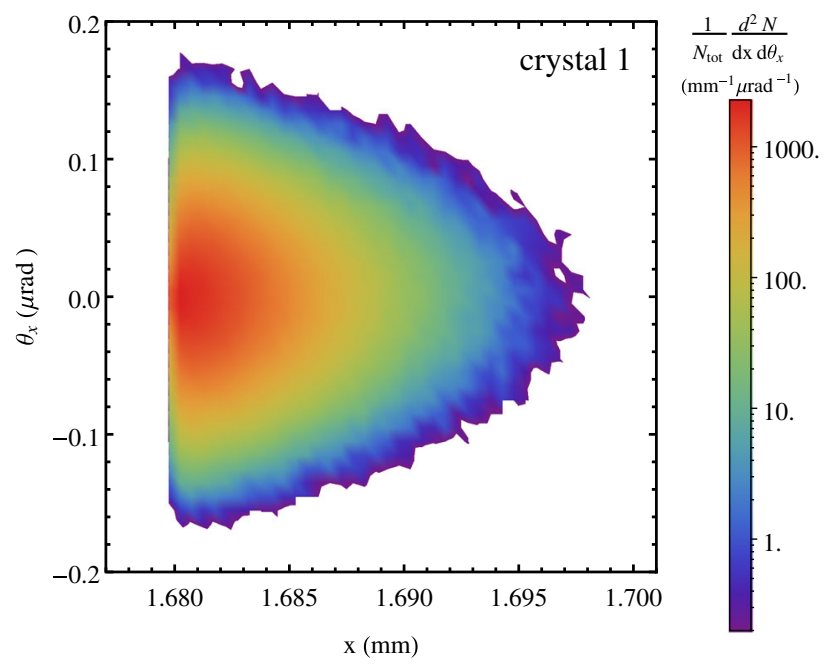

FIG. 12. Beam phase space at the first crystal entrance. 

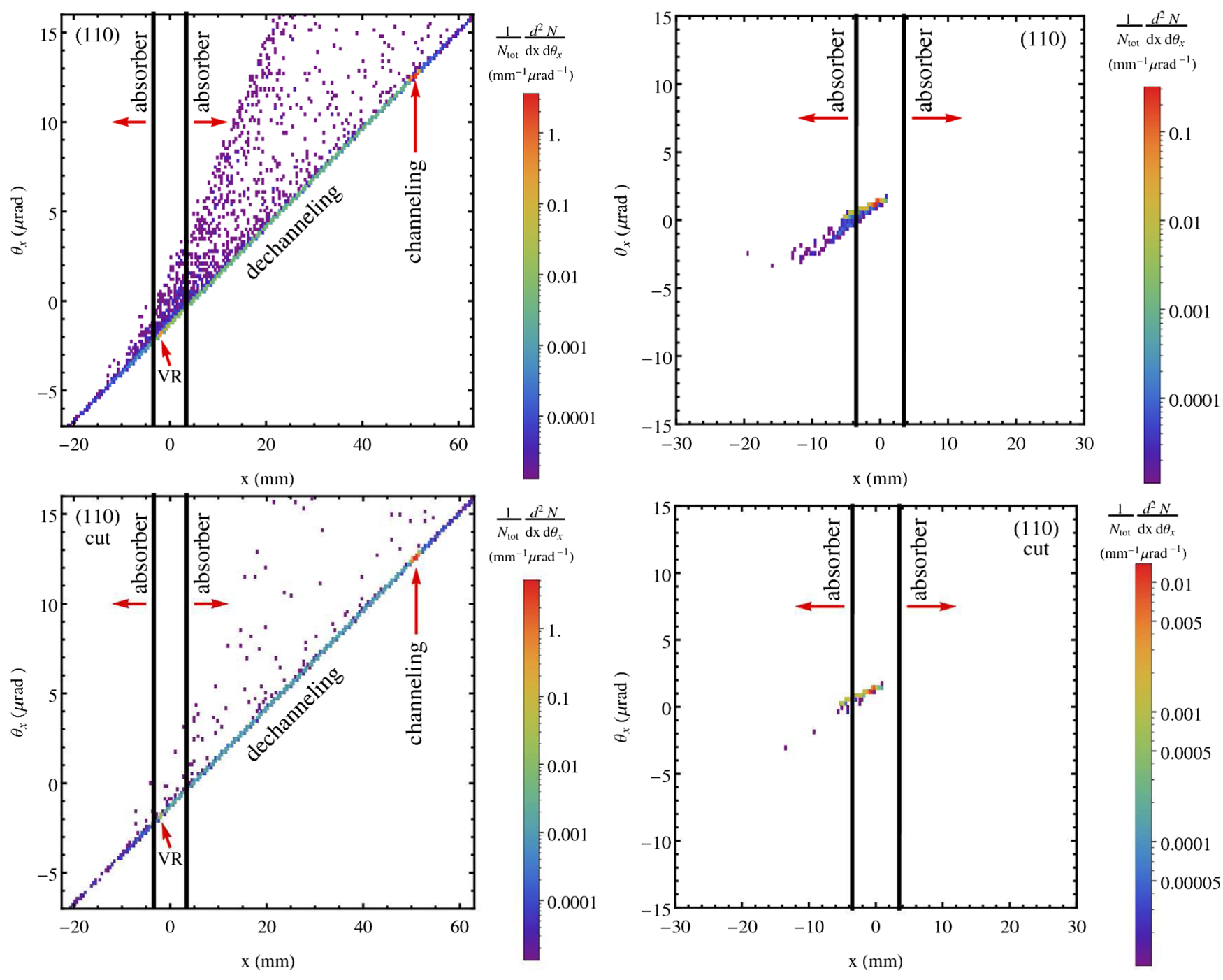

FIG. 13. Beam phase space, normalized to the total number of particles, passed through the cleaning insertion, at the position of the first (left) and the second (right) absorber for single crystal-based collimation system. The Si crystal the same as in Fig. 10, aligned for planar channeling in (110) vertical crystal planes without cut (top) and with cut (bottom). The crystal and the absorbers are placed at 7.2 and $12.6 \sigma$, respectively.

The beam phase space was generated at the longitudinal positions of the first absorber/second crystal and the second absorber for all the variants of collimation schemes, as shown in Figs. 13-14. In the latter, one can also easily distinguish the coherent effects deflecting the particles. In order to demonstrate the amount of particles, intercepted by the absorber, all phase spaces were normalized to the total number of particles, passed through the cleaning insertion $N_{\text {tot }}$.

The crystal with the cut, providing considerably higher channeling efficiency, demonstrates considerable reduction of particles, nonintercepted by the betatron cleaning insertion, namely from $9.6 \%$ (without the cut) down to $0.5 \%$ (with the cut). One can easily observe this result from the scale in the right column of Fig. 13 decreased on an order of magnitude for the crystal with the cut.

The double crystal-based collimation scheme also demonstrates a considerable decrease of nonintercepted beam fraction, namely down to $1.3 \%$ (see Fig. 14). Moreover, since the deflection angle of nonchanneled particles becomes considerably higher in the first crystal due to the MVROC effect, channeling in skew planes also enhances the efficiency of the single crystal-based collimation scheme. Additionally, the application of the cut in the first crystal of the double collimation system reduces the nonintercepted fraction on one more order of magnitude, down to $0.1 \%$.

Moreover, the impact parameters of particle incidence to absorbers are high enough to prevent particle leakage at the absorber boundaries. Therefore, the absorber length can be chosen high enough to intercept all the particles. For instance, for $1 \mathrm{~m}$ long tungsten absorber the leakage, caused by nonabsorbtion, is $\sim 4 \times 10^{-5}$, being $\sim 2 \times 10^{-9}$ for $2 \mathrm{~m}$ of tungsten, calculated using nuclear interaction length [33]. 

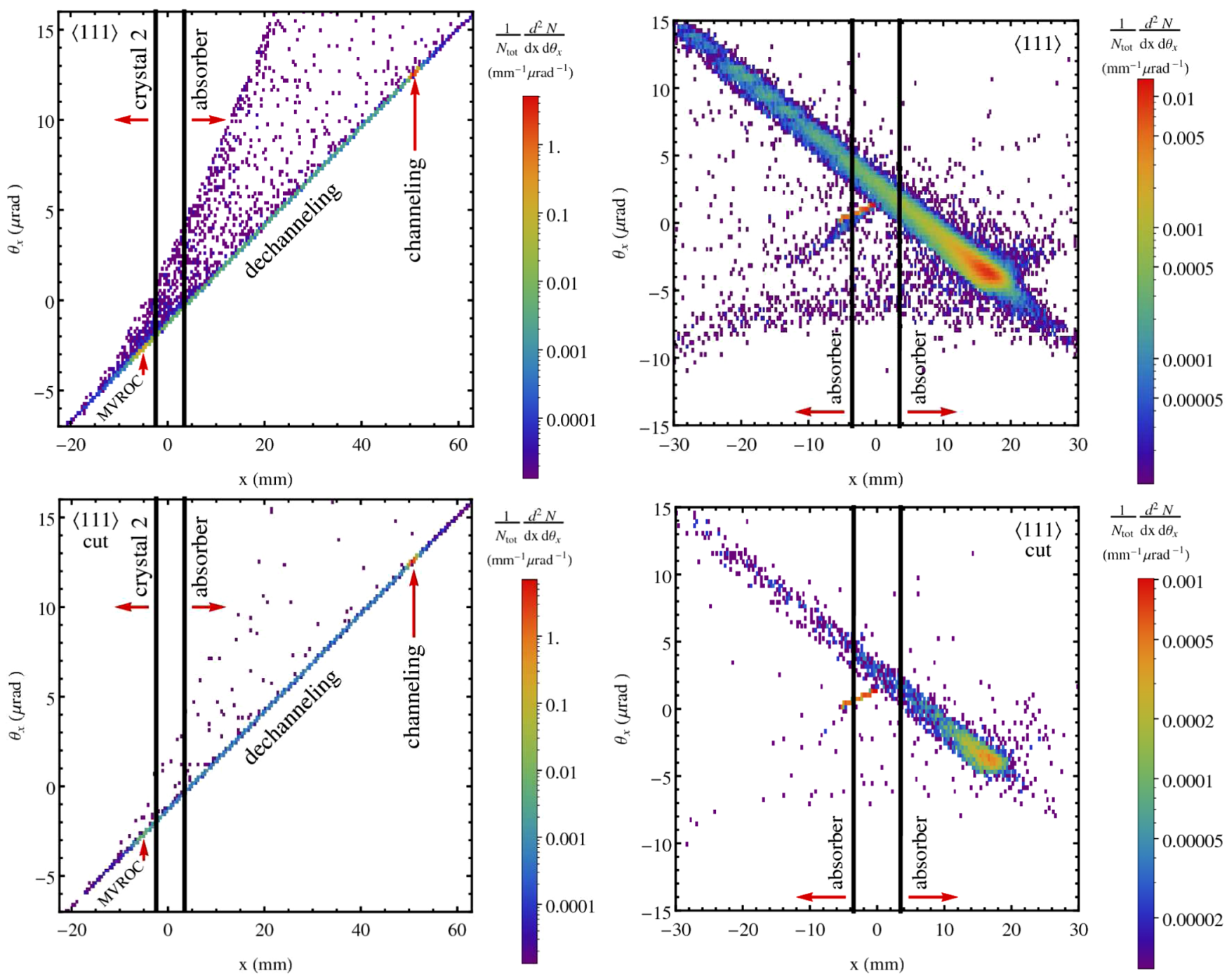

FIG. 14. Beam phase space, normalized to the total number of particles, passed through the cleaning insertion, at the position of the first (left) and the second (right) absorber for double crystal-based collimation system. The first Si crystal the same as in Fig. 10, aligned for planar channeling in skew (110) crystal planes, formed by $\langle 111\rangle$ axes, without the cut (top) and with the cut (bottom). The second Si crystal is the same as in Fig. 8, aligned for MVROC. The first and second crystal are placed at 7.2 and $9 \sigma$, respectively, as well as the absorbers are placed at $12.6 \sigma$.

Therefore, the double crystal-based collimation scheme as well as coherent effects proposed allow one to intercept approximately $99 \%$ of particles by only one passage through the betatron cleaning insertion. The application of the crystal cut in the double crystal-based collimation scheme increases this number up to $99.9 \%$.

\section{CONCLUSIONS}

The double crystal-based collimation system, combined with the layout of the betatron cleaning insertion of the Future Circular Collider [17], has been suggested, based on application of the second bent crystal to amplify the deflection angle of nonchanneled particles and to reduce, therefore, the leakage to sensitive equipment. The effects of multiple volume reflection in one bent crystal, volume reflection in a crystal sequence as well as MVROC in a crystal sequence have been proposed for the second crystal.

A theoretical model of optimization of crystal geometry and alignment has been proposed. It is mainly based on estimation of the dependence of the ratio of the volume reflection angle to the Lindhard angle on the ratio of the bending radius to its critical value, being independent on particle energy. By this model we have optimized the parameters of a bent crystal sequence for both volume reflection and MVROC at a fixed crystal number and length of the sequence. Monte Carlo simulations conducted for various lengths and crystal numbers has allowed us to choose the optimal crystal number for each length as well as to compare the peak deflection angle of volume reflection and MVROC. 
Volume reflection in a silicon crystal sequence provides higher deflection angle than MVROC (12 $\mu \mathrm{rad}$ and $8.3 \mu \mathrm{rad}$ at $10 \mathrm{~cm}$ of the length). However, MVROC in a silicon crystal sequence increases this angle up to $16 \mu \mathrm{rad}$. Additionally, it requires 3-4 crystals, while for volume reflection 13 crystals in a sequence are necessary at the given length. However, MVROC in heavy crystals allows one to reach deflection angles even higher, in particular $23 \mu \mathrm{rad}$ for the tungsten crystal.

For the first crystal in the double crystal-based collimation system channeling in skew planes have been suggested. On the one hand it five times increases the deflection angle of nonchanneled particles by means of application of MVROC instead of volume reflection. On the other hand it increases the angular acceptance and, therefore, the channeling efficiency, in particular from $79 \%$ up to $80.5 \%$ in the case simulated. In addition, the application of a crystal cut has been suggested allowing one to increase the channeling efficiency from $79 \%$ and $80.5 \%$ up to $87.5 \%$ and $89 \%$.

The double crystal-based collimation system as well as all the effects of particle deflection by a bent crystal listed above allow one to intercept approximately $99 \%$ of particles by only one passage through the betatron cleaning insertion at the FCC. The application of the crystal cut increases the intercepted fraction up to $99.9 \%$. Moreover, high impact parameters of particle incidence to absorbers prevent leakage at the absorber boundaries. This allows one to choose long enough absorbers for almost $100 \%$ absorbtion. Therefore the collimation schemes proposed are potentially applicable at high-energy accelerators and colliders, in particular the Future Circular Collider.

\section{ACKNOWLEDGMENTS}

We acknowledge the CINECA award under the ISCRA initiative for the availability of high performance computing resources and support.

[1] O. Brüning, P. Collier, P. Lebrun, S. Myers, R. Ostojic, J. Poole, and P. Proudlock, LHC Design report, Vol. I, The LHC Main Ring (CERN, Geneve, 2004).

[2] G. Apollinari, I. Béjar Alonso, O. Brüning, M. Lamont, and L. Rossi (HiLumi LHC Collaboration), Report No. CERN-ACC-2014-0300, 2014.

[3] S. Redaelli, in Proc. of the Joint International Accelerator School, Newport Beach, United States, 2014, edited by R. Schmidt (CERN, Geneva, 2016), p. 403.

[4] W. Scandale, Use of crystals for beam deflection in particle accelerators, Mod. Phys. Lett. A 27, 1230007 (2012).

[5] M. Maslov, N. Mokhov, and I. Yazynin, SSCL Report No. 484, 1991.

[6] D. Trbojevic, V. Biryukov, M. Harrison, B. Parker, P.Thompson, A. Stevens, N. Mokhov, and A. Drozhdin, in Proc. of 6th European Particle Accelerator Conf.,
Stockholm, Sweden, 1998, edited by L. Liljeby, S. Myers, Ch. Petit-Jean-Genaz, J. Poole, and K.-G. Rensfelt (Institute of Physics Publishing, Bristol and Philadelphia, 1998), p. 2146.

[7] A. Afonin, V. M. Biryukov, V. N. Chepegin, Yu. A. Chesnokov, V. I. Kotov, V. I. Terekhov, E. F. Troyanov, Yu. S. Fedotov, Yu. M. Ivanov, W. Scandale, and M. B. H. Breese, in Proc. of 7th ICFA mini-workshop, Lake Como 1999 (Fermi National Accelerator Laboratory, Batavia, 2000), pp. 127.

[8] A. G. Afonin, V. T. Baranov, S. Bellucci, S. A. Belov, S. Bini, V. N. Gorlov, G. Giannini, A. D. Ermolaev, I. V. Ivanova, D. M. Krylov, V. A. Maisheev, D. A. Savin, E. A. Syshchikov, V. I. Terekhov, V. N. Chepegin, Yu. A. Chesnokov, P. N. Chirkov, and I. A. Yazynin, Crystalassisted beam extraction and collimation at the U-70 circular accelerator, Instrum. Exp. Tech. 54, 1 (2011).

[9] Yu. A. Chesnokov, A. G. Afonin, V. T. Baranov, G. I. Britvich, P. N. Chirkov, V. A. Maisheev, V. I. Terekhov, and I. A. Yazynin, Bent crystal channeling applications for beam splitting, extraction and collimation in the U-70 accelerator of IHEP, Nucl. Instrum. Methods Phys. Res., Sect. B 309, 105 (2013).

[10] W. Scandale et al., First results on the SPS beam collimation with bent crystals, Phys. Lett. B 692, 78 (2010).

[11] W. Scandale et al., Comparative results on collimation of the SPS beam of protons and $\mathrm{Pb}$ ions with bent crystals, Phys. Lett. B 703, 547 (2011).

[12] W. Scandale et al., Strong reduction of the off-momentum halo in crystal assisted collimation of the SPS beam, Phys. Lett. B 714, 231 (2012).

[13] N. V. Mokhov et al., Crystal collimation studies at the tevatron (T-980), Int. J. Mod. Phys. A 25, 98 (2010).

[14] N. Mokhov, J. Annala, R. Carrigan, M. Church, A. Drozhdin, T. Johnson, R. Reilly, V. Shiltsev, G. Stancari, D. Still, A. Valishev, X.-L. Zhang, and V. Zvoda, Tevatron beam halo collimation system: design, operational experience and new methods, J. Instrum. 6, T08005 (2011).

[15] W. Scandale et al., Observation of channeling for 6500 $\mathrm{GeV} / \mathrm{c}$ protons in the crystal assisted collimation setup for LHC, Phys. Lett. B 758, 129 (2016).

[16] A. Taratin, "Volume reflection" of high-energy charged particles in quasi-channeling states in bent crystals, Phys. Lett. A 119, 425 (1987).

[17] M. Fiascaris, R. Bruce, D. Mirarchi, and S. Redaelli, in Proc. of IPAC2016, Busan, Korea, 2016 (CERN, Geneva, 2016), p. 2423.

[18] V. Tikhomirov, Multiple volume reflection from different planes inside one bent crystal, Phys. Lett. B 655, 217 (2007).

[19] V. Guidi, A. Mazzolari, and V. Tikhomirov, On the observation of multiple volume reflection from different planes inside one bent crystal, J. Appl. Phys. 107, 114908 (2010).

[20] W. Scandale et al., Multiple volume reflections of highenergy protons in a sequence of bent silicon crystals assisted by volume capture, Phys. Lett. B 688, 284 (2010).

[21] W. Scandale et al., First observation of multiple volume reflection by different planes in one bent silicon crystal for high-energy protons, Phys. Lett. B 682, 274 (2009). 
[22] A. G. Afonin, V. T. Baranov, M. K. Bulgakov, I. S. Voinov, V. N. Gorlov, I. V. Ivanova, D. M. Krylov, A. N. Lun'kov, V. A. Maisheev, S. F. Reshetnikov, D. A. Savin, E. A. Syshchikov, V. I. Terekhov, Yu. A. Chesnokov, P. N. Chirkov, and I. A. Yazynin, Collimation of the circulating beam in the U-70 synchrotron by means of the reflection of particles in crystals with axial orientation, JETP Lett. 93, 187 (2011).

[23] W. Scandale et al., Observation of multiple volume reflection by different planes in one bent silicon crystal for high-energy negative particles, Europhys. Lett. 93, 56002 (2011).

[24] M. Benedikt, D. Schulte, and F. Zimmermann, Optimizing integrated luminosity of future hadron colliders, Phys. Rev. ST Accel. Beams 18, 101002 (2015).

[25] M. Benedikt and F. Zimmermann, Towards Future Circular Colliders, J. Korean Phys. Soc. 69, 893 (2016).

[26] A. Ball, M. Benedikt, L. Bottura, O. Dominguez, F. Gianotti, B. Goddard, P. Lebrun, M. Mangano, D. Schulte, E. Shaposhnikova, R. Tomas, and F. Zimmermann, Future Circular Collider Study Hadron Collider Parameters (CERN, Geneve, 2014), https://indico.cern.ch/event/298180/ contributions/1658149/attachments/560575/772288/FCC1401101315-DSC_HadronColliderParameters_V0.3.pdf.

[27] https://impedance.web.cern.ch/impedance/fcchh/beam_ dynamics_parameters.html.

[28] V. Tikhomirov and A. Sytov, Multiple volume reflection in one crystal as an origin of significant scattering intensity and radiation power increase, Nucl. Instrum. Methods Phys. Res., Sect. B 309, 109 (2013).
[29] V. Tikhomirov, A technique to improve crystal channeling efficiency of charged particles, J. Instrum. 2, P08006 (2007).

[30] V. Guidi, A. Mazzolari, and V. Tikhomirov, Increase in probability of ion capture into the planar channelling regime by a buried oxide layer, J. Phys. D 42, 165301 (2009).

[31] V. A. Maisheev, Volume reflection of ultrarelativistic particles in single crystals, Phys. Rev. ST Accel. Beams 10, 084701 (2007).

[32] J. Lindhard, Influence of crystal lattice on motion of energetic charged particles, Mat. Fys. Medd. K. Dan. Vidensk. Selsk 34, 14 (1965).

[33] K. A. Olive et al. (Particle Data Group), Review of particle physics, Chin. Phys. C 38, 090001 (2014).

[34] A. Sytov, Application of a bent crystal for proton beam extraction from a storage ring, Vestnik of the Bel. St. Univ. Series 1, 48 (2014).

[35] A. Sytov and V. Tikhomirov, CRYSTAL simulation code and modeling of coherent effects in a bent crystal at the LHC, Nucl. Instrum. Methods Phys. Res., Sect. B 355, 383 (2015).

[36] V. V. Tikhomirov and A. I. Sytov, The miscut angle influence on the future LHC crystal based collimation system, Problems of Atomic Science and Technology 1, 8892 (2012).

[37] J. Molson, P. Bambade, S. Chancé, and A. Faus-Golfe, in Proc. of IPAC2016, Busan, Korea, 2016 (CERN, Geneva, 2016), p. 1381. 